\title{
Établissement de critères pour lancer des enquêtes sur les éclosions de maladies entériques en Colombie-Britannique
}

\author{
Taylor M. ${ }^{1^{*}}$ et Galanis E. ${ }^{1,2}$ \\ Centre de contrôle des maladies de la Colombie-Britannique, Vancouver (C.-B.) \\ École de santé publique et de santé des populations, Université de la Colombie-Britannique, Vancouver (C.-B.) \\ Auteure-ressource : marsha.taylor@bccdc.ca
}

\section{Résumé}

Objectif : Établir et évaluer les critères pour lancer des enquêtes sur les éclosions de maladies entériques à l'échelle provinciale en fonction des caractéristiques des alertes, des grappes de cas et des enquêtes réussies sur les éclosions.

Méthodes : Nous avons effectué le suivi de toutes les alertes de maladies entériques et de grappes de cas signalées au Centre de contrôle des maladies de la Colombie-Britannique en 2011 et 2012. Des renseignements ont été recueillis sur l'étiologie, le nombre de cas, la répartition géographique, les dates signalées et la méthode de déclaration. Les mesures ont été classées en trois catégories : aucune mesure supplémentaire, examen/rapport ou enquête. Les résultats des enquêtes sur les éclosions ont été classés en deux catégories : résolue et non résolue. Les données de 2011 ont été utilisées pour déterminer les caractéristiques des alertes et des grappes de cas plus susceptibles de mener à des enquêtes sur les éclosions et les caractéristiques des éclosions résolues dans le but d'établir des critères. Les critères pour lancer une enquête sur l'éclosion ont été évalués rétrospectivement à l'aide des données de 2011, puis mis en œuvre en 2012.

Résultats : En 2011, 251 alertes ou grappes de cas de maladies entériques ont été signalées. Quatorze (5,6\%) ont entraîné une enquête sur l'éclosion et neuf (64,3\%) éclosions ont été résolues. En analysant les données de façon rétrospective, on a déterminé des critères à partir des alertes et des grappes de cas ayant mené à des enquêtes réussies sur les éclosions : spécificité du pathogène, déclaration en temps opportun, source commune ou événement commun, éclosions multirégionales ou éclosions signalées par d'autres organismes. Après avoir appliqué ces critères de façon prospective en 2012, nous avons pris des mesures sur une plus petite proportion des 244 alertes et grappes de cas (32\% par rapport à 44,6\% en 2011) et 66,7\% d'entre elles ont été résolues (comparativement à 64,3\% en 2011).

Conclusion : L'évaluation continue déterminera si cela permettra d'améliorer les résultats des enquêtes sur les éclosions et l'utilisation des ressources en Colombie-Britannique.

\section{Introduction}

L'objectif des enquêtes sur les éclosions de maladies entériques (y compris les infections d'origine alimentaire, hydrique et celles qui se transmettent par contact de personne à personne ou à animal) est de déterminer la source de la maladie afin de lutter contre l'éclosion, de limiter le nombre de cas et de formuler des recommandations dans le but de prévenir de futures éclosions. La détermination d'une source fournit également des renseignements sur les produits à risque élevé, les modes de transmission et les interventions efficaces (1). Toutefois, la proportion d'éclosions de maladies entériques résolues (où une source est déterminée) est faible $(2,3)$. En Colombie-Britannique (C.-B.), nous avons déjà pris des mesures en vue d'améliorer les enquêtes sur les éclosions en déterminant les maladies entériques devant faire systématiquement l'objet d'une enquête, en utilisant des formulaires normalisés d'enquête de cas et en maintenant des relations de collaboration entre les épidémiologistes et le personnel de laboratoire.

Les enquêtes sur les éclosions sont coûteuses et exigent beaucoup de ressources. La réponse aux alertes qui ne se transforment pas en éclosions (fausses alarmes) et les enquêtes infructueuses entraînent un gaspillage des ressources limitées en matière de santé publique. L'amélioration de la capacité à résoudre et à contrôler les 
éclosions en déterminant celles qui ont la plus grande possibilité de succès et en se concentrant sur ces dernières, de façon à ce que les ressources puissent être utilisées plus adéquatement, serait utile aux professionnels de la santé publique. En outre, l'utilisation d'une approche uniforme et transparente pour lancer des enquêtes sur les éclosions crée une norme de pratique et augmente la confiance des partenaires dans le processus.

En Colombie-Britannique, où la population est de 4,5 millions de personnes (4), le Centre de contrôle des maladies de la Colombie-Britannique est responsable de la surveillance à l'échelle provinciale et de la coordination des enquêtes sur les éclosions qui touchent plusieurs régions sanitaires locales. Le Centre participe également aux enquêtes sur les éclosions à l'échelle nationale et offre de l'aide en cas d'éclosions dans une seule région sanitaire locale, au besoin. En 2009 et 2010, il y a eu une moyenne de 225 alertes et grappes de cas de maladies entériques signalées chaque année au Centre; 10 des 19 (52,6\%) enquêtes sur les éclosions entreprises ont été résolues. Au cours de cette période, il n'existait pas de critères pour amorcer une enquête et la décision de lancer une enquête était prise en fonction de l'expérience et du jugement des enquêteurs. À notre connaissance, on n'utilise pas de critères au Canada pour lancer des enquêtes sur les éclosions de maladies entériques.

Les objectifs de ce travail étaient les suivants : 1) déterminer les caractéristiques des alertes et des grappes de cas de maladies entériques que nous avons analysées; 2 ) déterminer les caractéristiques des enquêtes réussies sur les éclosions de maladies entériques en 2011 afin d'établir des critères pour lancer des enquêtes sur les éclosions de maladies entériques à l'échelle provinciale; et 3) évaluer ces critères et comparer les enquêtes sur les éclosions en 2011 et 2012.

\section{Méthodes}

Un processus systématique a été utilisé pour effectuer le suivi de toutes les déclarations d'alertes et de grappes de cas de maladies entériques reçues de diverses sources entre le $1^{\text {er }}$ janvier 2011 et le 31 décembre 2012. On a inclus les alertes de deux cas ou plus causés par le même pathogène ainsi que les grappes de cas dans lesquels deux cas ou plus non semblables présentant des maladies similaires étaient liés sur le plan épidémiologique. La seule exception à ces critères était le botulisme, pour lequel un cas a été considéré comme étant une éclosion, et I'hépatite A, qui a été exclue puisqu'elle fait l'objet d'une enquête par un autre groupe du Centre de contrôle des maladies de la Colombie-Britannique. Toutes les grappes de cas et les alertes devaient avoir été signalées aux épidémiologistes du Centre ou déterminées par ces derniers. Les sources des grappes de cas signalées comprenaient les régions sanitaires locales, le Laboratoire de santé publique et de référence en microbiologie de la Colombie-Britannique, les autres provinces et territoires, les organismes fédéraux ( $p$. ex. l'Agence canadienne d'inspection des aliments, l'Agence de la santé publique du Canada) et les organismes internationaux (p. ex. les ministères des services de santé d'état aux États-Unis).

La source des alertes était la base de données provinciale des maladies à déclaration obligatoire. Le Centre de contrôle des maladies de la Colombie-Britannique utilise un système de détection automatique des aberrations sur les maladies à déclaration obligatoire chaque semaine. Les données saisies comprennent cinq ans de données sur les maladies à déclaration obligatoire à l'échelle du genre et cinq ans de données de laboratoire (à l'échelle du sérotype, de l'espèce et du lysotype ( $S$. enteritidis, $S$. Heidelberg) ou d'électrophorèse en champ pulsé (E. coli O157, S. typhimurium, S. sonnei)). Une analyse de séries chronologiques détermine chaque semaine les aberrations (alertes) en tenant compte des tendances saisonnières et temporelles. Les alertes sont produites lorsque le nombre de cas de la maladie est nettement supérieur à celui prévu au cours de périodes de $7,14,21$ et 28 jours, à l'échelle provinciale ou locale.

Des renseignements sur les alertes et les grappes de cas ont été recueillis de façon prospective au moment de la déclaration. Chaque alerte ou grappe de cas qui répondait aux critères d'inclusion a été prise en compte et des renseignements ont été recueillis sur l'étiologie, y compris le niveau de sous-typage, le nombre de cas, la répartition géographique, la date de déclaration initiale, la connaissance préalable d'alerte ou de grappe et la méthode de déclaration initiale. En outre, les mesures prises par un épidémiologiste du Centre de contrôle des maladies de la Colombie-Britannique pour chaque alerte ou grappe de cas ont été consignées. Les mesures ont été classées en trois catégories: 
- Aucune autre mesure prise.

- Examen/Rapport - Nous avons utilisé des données sur les maladies à déclaration obligatoire ou des données de laboratoire existantes pour évaluer les points communs ou nous avons transmis les renseignements aux partenaires de santé publique, sans exiger d'enquête plus approfondie.

- Enquête sur l'éclosion - Nous avons utilisé une approche coordonnée pour mener une enquête sur une éclosion ou y répondre. En général, ces mesures comprennent la demande et l'examen des renseignements en matière d'exposition des cas des régions sanitaires locales ainsi que l'utilisation de méthodes d'enquête supplémentaires (p. ex. nouvelles entrevues, enquêtes de site, échantillonnage environnemental) afin de déterminer et de contrôler la source.

Parmi les alertes et les grappes de cas ayant mené à une enquête sur l'éclosion, nous avons classé comme résolues celles dont la source était déterminée à partir d'une étude analytique, d'une détection du pathogène en laboratoire ou d'une combinaison de renseignements en matière d'exposition des cas et de retraçage à une possible source commune.

Les données de 2011 ont été utilisées afin d'établir des critères pour lancer une enquête sur l'éclosion en fonction des renseignements sur le pathogène, le nombre de cas, la période entre la déclaration et le plus récent cas signalé en utilisant la première des dates disponibles (date de déclaration, date de prélèvement ou date d'apparition des symptômes), la géographie et les renseignements disponibles en matière d'exposition. Les critères ont été établis en comparant les alertes et les grappes de cas ayant mené à une enquête sur l'éclosion à celles n'y ayant pas mené, ainsi que les enquêtes sur l'éclosion résolues à celles non résolues. Les données de 2011 ont été utilisées pour évaluer les critères de façon rétrospective. Les critères ont été utilisés tout au long de l'année 2012 et les données ont servi à évaluer les critères de façon prospective en comparant le nombre et la proportion des alertes et des grappes de cas qui ont fait l'objet d'enquêtes, qui ont été résolues et contrôlées par rapport à 2011 pour évaluer les répercussions de la mise en place des critères. On a utilisé le logiciel MS Excel ${ }^{\circledR}$ pour la collecte et l'analyse des données. Les tests du chi carré ont été calculés à l'aide des logiciels SAS et Epi Calc 2000 pour comparer les proportions et les médianes. Une valeur $p<0,05$ était considérée comme significative.

\section{Résultats \\ Évaluation des alertes, des grappes de cas et des enquêtes}

En 2011, un total de 251 alertes et grappes de cas ont été signalées. Quatorze $(5,6)$ ont mené à une enquête sur l'éclosion (Tableau 1). Les alertes et les grappes de cas qui ont mené à une enquête sur l'éclosion étaient significativement différentes de celles qui n'ont pas mené à une enquête; elles présentaient une proportion plus élevée d'électrophorèse en champ pulsé $(p=<0,001)$, elles étaient plus susceptibles de toucher de multiples régions sanitaires locales $(p=0,002)$ et une proportion plus élevée de ces alertes et grappes de cas avaient été signalées par d'autres organismes $(p=<0,001)$. Le nombre médian de cas était plus élevé, bien que non significatif, et la période entre le cas le plus récent et la déclaration était semblable (Tableau 1). 
Tableau 1. Caractéristiques des alertes et des grappes de cas de maladies entériques signalées au Centre de contrôle des maladies de la Colombie-Britannique, par type de mesures prises,

Colombie-Britannique, 2011 ( $n=251$ )

\begin{tabular}{|l|c|l|c|c|}
\hline $\begin{array}{l}\text { Caractéristiques au } \\
\text { moment de la } \\
\text { déclaration }\end{array}$ & $\begin{array}{l}\text { Enquête menée } \\
\text { sur l'éclosion } \\
(\mathbf{n}=\mathbf{1 4})\end{array}$ & $\begin{array}{l}\text { Données } \\
\text { examinées ou } \\
\text { rapports aux } \\
\text { partenaires } \\
(\mathbf{n}=\mathbf{9 8})\end{array}$ & $\begin{array}{l}\text { Aucune } \\
\text { autre mesure } \\
\text { prise }\end{array}$ & Valeur $\mathbf{p}$ \\
$\mathbf{( n = 1 3 9 )}$ & \\
\hline Proportion du total & $5,6 \%$ & $39,0 \%$ & $55,4 \%$ & Sans objet \\
\hline $\begin{array}{l}\text { Nombre (\%) avec } \\
\text { électrophorèse en } \\
\text { champ pulsé (PFGE) }\end{array}$ & $6(42,8 \%)$ & $13(13,3 \%)$ & $4(2,9 \%)$ & $<0,001$ \\
\hline $\begin{array}{l}\text { Nombre médian de } \\
\text { cas; intervalle }\end{array}$ & $6(1-30)$ & $3(1-106)$ & $3(1-68)$ & 0,630 \\
\hline $\begin{array}{l}\text { Nombre médian (et } \\
\text { intervalle) de jours } \\
\text { entre la déclaration et } \\
\text { le plus récent cas } \\
\text { déclaré }\end{array}$ & $4(1-30)$ & $3(1-47)$ & Non évalué & 0,710 \\
\hline $\begin{array}{l}\text { Nombre (\%) touchant } \\
\text { plusieurs régions } \\
\text { sanitaires locales }\end{array}$ & $5(35,7 \%)$ & $22(22,4 \%)$ & $15(10,8 \%)$ & 0,002 \\
\hline $\begin{array}{l}\text { Nombre (\%) déclaré } \\
\text { par un autre } \\
\text { organisme }\end{array}$ & $10(71,4 \%)$ & $14(14,3 \%)$ & 0 & $<0,001$ \\
\hline
\end{tabular}

En 2011, neuf (64,3\%) des éclosions ont été résolues. Les éclosions résolues étaient plus susceptibles de comprendre un pathogène/sous-type rare, d'avoir été déclarées par un autre organisme et de compter moins de deux semaines entre le cas le plus récent et la déclaration. Aucune de ces différences n'était statistiquement significative. Un événement/emplacement commun et l'indication d'une source au moment de la déclaration n'ont été déterminés que dans les enquêtes résolues (Tableau 2). 
Tableau 2. Caractéristiques des enquêtes sur les éclosions de maladies entériques résolues et non résolues, Colombie-Britannique, $2011(n=14)$

\begin{tabular}{|c|c|c|}
\hline Caractéristiques au moment de la déclaration & $\begin{array}{l}\text { Éclosion } \\
\text { résolue* } \\
(n=9)\end{array}$ & $\begin{array}{l}\text { Éclosion non } \\
\text { résolue } \\
(n=5)\end{array}$ \\
\hline Proportion du total & $64,3 \%$ & $35,7 \%$ \\
\hline Spécificité du pathogène & & \\
\hline $\begin{array}{l}\text { Nombre et \% avec électrophorèse en champ pulsé } \\
\text { (PFGE) }\end{array}$ & $2(22,2 \%)$ & $4(80,0 \%)$ \\
\hline Nombre et \% possédant un pathogène/sous-type rare & $6(66,7 \%)$ & $2(40,0 \%)$ \\
\hline Réponse en temps opportun & & \\
\hline $\begin{array}{l}\text { Nombre médian (et intervalle) de jours entre la } \\
\text { déclaration et le plus récent cas déclaré }\end{array}$ & $3(1-30)$ & $16(1-21)$ \\
\hline $\begin{array}{l}\text { Nombre }(\%) \text { comptant moins de deux semaines entre } \\
\text { l'apparition, le rapport et la collecte du dernier cas et la } \\
\text { déclaration }\end{array}$ & $8(88,9 \%)$ & $2(40,0 \%)$ \\
\hline Nombre médian de cas; (intervalle) & $5(1-30)$ & $6(1-9)$ \\
\hline $\begin{array}{l}\text { Nombre (\%) touchant plusieurs régions sanitaires } \\
\text { locales }\end{array}$ & $3(33,3 \%)$. & $2(40,0 \%)$ \\
\hline Nombre (\%) déclaré par un autre organisme & $7(77,8 \%)$ & $3(60,0 \%)$ \\
\hline Nombre (\%) avec une indication de la source & $4(44,4 \%)$ & 0 \\
\hline $\begin{array}{l}\text { Nombre (\%) ayant en commun un } \\
\text { événement/emplacement }\end{array}$ & $4(44,4 \%)$ & 0 \\
\hline
\end{tabular}

* Les éclosions résolues comprenaient : l'intoxication par phycotoxine diarrhéique associée à des moules cuites (5), le botulisme associé à de la gelée de melon d'eau; l'infection à E. coli O157:H7 associée au foie de veau; l'infection à S. kingabwa associée aux lézards de compagnie; l'infection à $S$. enteritidis associée aux œufs; deux enquêtes sur le norovirus dans un centre de villégiature; l'infection à $S$. agbeni associée à un manipulateur d'aliments (6); l'infection à $S$. infantis associée à un manipulateur d'aliments.

** Les éclosions non résolues comprenaient : une enquête sur l'infection à Campylobacter au sein de la collectivité; une enquête sur l'infection à E. coli O157:H7 au sein de la collectivité; et trois enquêtes sur l'infection à S. enteritidis au sein de la collectivité.

\section{Établissement de critères}

Des critères propres à chaque pathogène ont été établis pour déterminer les alertes et les grappes de cas à examiner et devant faire l'objet d'une enquête (Tableau 3). Les critères comprennent un nombre minimal de cas selon le pathogène et la spécificité, une période maximale entre l'apparition du cas le plus récent et la déclaration, l'exigence d'une distribution multirégionale ou d'un lien épidémiologique, ou encore des enquêtes amorcées par d'autres organismes. 
Figure 1. Critères pour l'examen des alertes et des grappes de cas de maladies entériques à l'échelle provinciale par le Centre de contrôle des maladies de la Colombie-Britannique ${ }^{1}$

\begin{tabular}{|c|c|c|}
\hline $\begin{array}{l}\text { Nombre minimal de } \\
\text { cas }\end{array}$ & \multicolumn{2}{|c|}{$\begin{array}{l}\text { Pathogène de sérotype commun ou d'espèce commune avec d'autres } \\
\text { typages, ou sérotype ou espèce rare (p. ex. E. coli O157 avec les mêmes } \\
\text { résultats d'électrophorèse en champ pulsé, Listeria monocytogenes) : } \\
\text { Étendue de } 2 \text { à } 5 \text { cas } \\
\text { Pathogène de genre/sérotype commun ou d'espèce commune sans autre } \\
\text { typage (p. ex. S. heidelberg sans typage moléculaire, } \\
\text { Campylobacter jejuni, Giardia) : Étendue de } 10 \text { à } 20 \text { cas } \\
\text { ET }\end{array}$} \\
\hline Période maximale & \multicolumn{2}{|c|}{$\begin{array}{l}\text { Première des dates disponibles (date de rapport, date de collecte ou date } \\
\text { d'apparition des symptômes) du plus récent cas dans les } 14 \text { jours suivant } \\
\text { la date de déclaration } \\
\text { ET }\end{array}$} \\
\hline $\begin{array}{l}\text { Répartition } \\
\text { géographique }\end{array}$ & \multicolumn{2}{|l|}{ Multirégionale ou inconnue } \\
\hline \multicolumn{3}{|c|}{$\begin{array}{l}\text { OU Un seul cas de botulisme OU } \\
\text { - N'importe quel nombre de cas à la première des dates disponibles (date de rapport, date de } \\
\text { collecte ou date d'apparition de la maladie) dans les } 14 \text { jours suivant la date de déclaration } \\
\text { présentant un lien épidémiologique entre eux, une source commune ou un aliment commun } \\
\text { OU } \\
\text { - N'importe quel nombre de cas associés à des enquêtes amorcées par d'autres territoires de } \\
\text { compétence }\end{array}$} \\
\hline & $\begin{array}{l}\text { PPORT/ ENQUÊTE : } \\
\text { niveau d'une enquête est fondé sur le } \\
\text { hogène et les renseignements disponibles. }\end{array}$ & $\begin{array}{l}\text { EXCLUSION : } \\
\text { Les alertes ou les } \\
\text { grappes de cas } \\
\text { associées aux voyages } \\
\text { ou à l'immigration, à une } \\
\text { seule région sanitaire, à } \\
\text { une erreur de saisie de } \\
\text { données ou à une } \\
\text { enquête précédente }^{2}\end{array}$ \\
\hline
\end{tabular}

${ }^{1}$ Remarque : Les critères sont assujettis à des modifications au fil du temps dans le cadre du travail et de l'évaluation en cours. ${ }^{2} \mathrm{Si}$ on obtenait de nouveaux renseignements sur les mêmes cas ou si de nouveaux cas étaient signalés, les critères seraient appliqués en utilisant le nouveau niveau de renseignements.

Le nombre de cas parmi les alertes et les grappes de cas faisant l'objet d'une enquête et les éclosions résolues variait selon le pathogène. La spécificité du pathogène a aidé à la détermination et à la résolution des éclosions. Le niveau de spécificité requis variait selon le pathogène, tandis que les pathogènes communs nécessitaient le sérotype ou l'électrophorèse en champ pulsé (p. ex. E. coli 0157 et $S$. enteritidis), les pathogènes plus rares ou les syndromes n'en nécessitaient pas (p. ex. intoxication par phycotoxine diarrhéique). Nous avons fixé des seuils pour le nombre minimum de cas nécessaires au moment de la déclaration en tenant compte des niveaux de 
spécificité qui ont varié entre 2 (p. ex. la listériose) et 20 (p. ex. la lambliase). Pour les pathogènes ayant une plus grande spécificité, le nombre minimal de cas requis était plus faible.

Parmi les enquêtes réussies sur les éclosions en 2011 , sept $(77,8 \%)$ comptaient moins de sept jours entre l'apparition des symptômes chez le cas le plus récent et la déclaration (données non indiquées) et huit $(88,9 \%)$ comptaient moins de quatorze jours. Nous avons choisi un critère de période de 14 jours pour inclure la plupart des éclosions pouvant être résolues tout en reconnaissant la nécessité que des mesures soient prises en temps opportun.

Pour respecter le mandat provincial du Centre de contrôle des maladies de la Colombie-Britannique, nos critères devaient exiger que les alertes et les grappes de cas touchent plusieurs régions sanitaires locales ou aient une répartition géographique inconnue au moment de la déclaration.

Les éclosions résolues comprenaient toutes une indication de la source au moment de la déclaration et des liens épidémiologiques entre les cas ou un événement ou un emplacement commun. II est probable que ces caractéristiques indiquent également un regroupement temporel des cas et une déclaration potentiellement plus opportune.

\section{Évaluation des critères}

Les 14 enquêtes sur les éclosions de 2011 ont été évaluées à l'aide des critères. Onze (78,6 \%) éclosions ont satisfait aux critères pour une enquête plus poussée. Des trois qui n'y satisfaisaient pas, une enquête a été menée dans une seule région sanitaire locale et pour ce qui est des deux autres enquêtes, les plus récents cas étaient survenus avant la période de 14 jours précédant la déclaration ( 28 et 30 jours). L'enquête dans une seule région sanitaire locale a été résolue. Aucune des éclosions présentant une plus longue période n'a été résolue, ce qui indique que nos critères excluraient de façon appropriée de tels événements. En outre, deux événements qui n'ont pas fait l'objet d'une enquête en 2011 auraient satisfait aux critères de lancement d'une enquête. Les deux événements avaient été causés par la bactérie Salmonella (l'un avec un sérotype rare, l'autre avec un sérotype commun et présentant un appariement de l'électrophorèse en champ pulsé) et comptaient quatre cas signalés au cours d'une période de sept et de quatorze jours. L'examen rétrospectif des données de laboratoire et des renseignements sur les cas disponibles n'a pas permis de déterminer les points communs.

En 2012, les critères ont été mis en œuvre de façon prospective. Un total de 244 alertes et grappes de cas ont été signalées. Seules trois (1,2\%) ont mené à des enquêtes sur les éclosions; deux ont été résolues. En 2012 , un moins grand nombre d'alertes et de grappes de cas a donné lieu à un examen ou à un rapport (30,7\% comparativement à $39,0 \%$ ) et une plus grande proportion d'entre elles n'ont nécessité la prise d'aucune autre mesure (68,0 \% comparativement à 55,4\%) qu'en 2011. Aucune éclosion connue n'est passée inaperçue.

Tout au long de l'année 2012, des ajustements ont été apportés aux critères parce que de nouvelles méthodes de laboratoire (p. ex. modification de détection de la toxine de Shiga) ont été mises en œuvre, les pathogènes qui n'étaient pas déjà pris en considération ont été déterminés et certains seuils des critères ont été jugés trop sensibles.

\section{Discussion}

Les critères permettant de déterminer les éclosions qui étaient plus susceptibles d'être résolues comprenaient les caractéristiques de spécificité du pathogène, la réponse en temps opportun et les liens connus entre les cas ou une source possible au moment de la déclaration. L'utilisation de nos critères en 2012 a entraîné une diminution de $21,3 \%$ du nombre de grappes de cas et d'alertes qui ont été examinées. Cette diminution est en grande partie attribuable au fait d'avoir cessé d'examiner les alertes ne touchant qu'une seule région sanitaire locale $(48,4 \%)$. Cela a entraîné une réduction concomitante de l'utilisation des ressources tant à l'échelle provinciale que locale, étant donné que moins de temps a été consacré à localiser, à examiner, à partager et à analyser les données de façon centralisée. L'évaluation rétrospective de nos critères a permis de déterminer deux grappes de cas en 2011 qui répondaient à nos critères, mais qui n'ont pas fait l'objet d'une enquête, probablement en raison de renseignements limités sur les cas indiquant l'existence d'un rapprochement. 
Certains de nos critères sont compatibles avec ceux utilisés par d'autres territoires de compétence et certains sont uniques.

Au Minnesota, une évaluation des éclosions d'infection à Salmonella a obtenu la meilleure réussite avec trois cas signalés à l'agence de la santé publique de l'état dans une période de sept jours (7). Dans une évaluation similaire des éclosions d'infection à E. coli $0157: \mathrm{H7}$, plus la période au cours de laquelle les deux premiers isolats ont été reçus était courte, plus la probabilité de résolution de l'éclosion était grande. Même si notre mesure de temps était différente, cela indique que le fait d'avoir un nombre suffisant de cas signalés de façon rapprochée et récemment déclarés à une agence de la santé publique aide à déterminer les éclosions pouvant être résolues. Cela peut également indiquer un événement ou un emplacement commun. Des évaluations axées sur un seul pathogène ont démontré un plus grand succès dans l'enquête au moyen de l'électrophorèse en champ pulsé sur les grappes de cas d'infection à Salmonella et à E. coli O157:H7 comptant quatre cas ou plus et trois cas ou plus, respectivement $(7,8)$. Pour les grappes de cas d'infection à Salmonella $(n=3)$ déterminés par électrophorèse en champ pulsé et d'infection à $E$. coli produisant la toxine de Shiga $(n=3)$, le nombre de cas nécessaires en Colombie-Britannique était similaire à celui déterminé par Rounds et al. $(7,8)$. On a démontré que l'utilisation d'une méthode de sous-typage des pathogènes, comme l'électrophorèse en champ pulsé, est utile à la détection des éclosions, particulièrement les éclosions dispersées (2,7). L'incorporation des renseignements en matière de spécificité du pathogène dans nos critères est importante pour la détection des éclosions à l'échelle provinciale qui sont souvent plus dispersées.

D'autres évaluations similaires comprenaient un examen rétrospectif des grappes de cas au moyen de données de laboratoire ou des éclosions signalées à un système de surveillance électronique ou résumées par le territoire de compétence concerné (7-9). Notre méthode est unique dans la mesure où elle comprend la collecte prospective des données disponibles au moment de la déclaration pour documenter les décisions et établir des critères qui pourraient être utilisés et utiles à ce stade du processus d'évaluation. Notre proportion d'enquêtes résolues est supérieure ou similaire à celle d'autres exemples publiés (2,3,7-9) probablement parce que nous n'avons inclus que des grappes de cas et des alertes que nous avons classées comme des éclosions après les premières étapes d'examen, ou selon notre classification d'éclosions résolues.

Cette étude comporte un certain nombre de limites. Une méthode prospective fournissait un moins grand nombre d'années de données pour l'évaluation. Comme nous connaissions notre processus, nous avions la capacité de modifier nos actions au fil du temps. Cette connaissance peut avoir entraîné un biais dans la sélection des grappes de cas et des alertes plus susceptibles d'être résolues avant l'établissement de critères. Même si cela touche notre évaluation de la proportion d'éclosions résolues, cela ne devrait pas avoir d'incidence sur la détermination des critères pour le lancement d'une enquête. Nos critères sont susceptibles de déterminer les éclosions associées aux sources ponctuelles aiguës, aux sources continues et aux infections à transmission interhumaine dans un emplacement commun. II est possible que nos critères ne permettent pas de détecter de petites éclosions ou des éclosions intermittentes comprenant des cas qui se produisent sur une longue période. II nous faudra d'autres méthodes de déclaration pour les détecter et nous nous reposerons sur le sous-typage des pathogènes pour déterminer les liens. Nos critères peuvent être pertinents pour d'autres territoires de compétence, mais les niveaux des critères peuvent avoir à être ajustés en fonction de la disponibilité des renseignements sur les cas, de la volonté et de la disponibilité des ressources disponibles pour l'enquête ainsi que de la capacité de sous-typage en laboratoire.

Des efforts futurs seront entrepris pour mieux élaborer et préciser ces critères. Les critères doivent être souples afin que des modifications puissent être facilement apportées au fil du temps. La collecte et l'analyse continue des données sont prévues de façon à évaluer la proportion d'enquêtes résolues et contrôlées au fil du temps, afin de permettre des mises à jour des critères et de déterminer si des éclosions passent inaperçues.

Nous avons entrepris ce travail afin d'améliorer le processus d'enquête en cas d'éclosion et ses résultats. Ce processus nous a permis de mieux comprendre les éléments qui peuvent mener à la réussite des enquêtes en cas d'éclosions. Au fil du temps, nous espérons être en mesure d'améliorer la proportion d'éclosions résolues et contrôlées afin de réduire la morbidité associée à des maladies entériques et d'améliorer l'utilisation des ressources. 


\section{Références}

(1) Gould LH, Walsh KA, Vieira AR, Herman K, Williams IT, Hall AJ, Cole D. Surveillance for foodborne disease outbreaks-United States, 1998-2008. MMWR. 2013; 62(2):1-34

(2) Murphee R, Garman K, Phan Q, Everstine K, Gould LH, Jones, TF. Characteristics of foodborne disease outbreak investigations conducted by Foodborne Diseases Active Surveillance Network (FoodNet) sites, 2003-2008. Clin Infect Dis. 2012; 54(S5):S498-503.

(3) Jones TF, Rosenberg L, Kubota K, Ingram LA. Variability among states in investigating foodborne disease outbreaks. J Food Prot. 2013; 10(1):69-73.

(4) Government of British Columbia. BC Stats-Population Estimates. Accès : http://www.bcstats.gov.bc.ca/StatisticsBySubject/Demography/PopulationEstimates.aspx

(5) Taylor M, McIntyre L, Ritson M, Stone J, Bronson R, Bitzikos O, Rourke W, Galanis E, Outbreak Investigation Team. Outbreak of diarrhetic shellfish poisoning associated with mussels, British Columbia, Canada. Mar Drugs. 2013; 15(5):1669-76.

(6) Taylor M, Brisdon S, Jeyes J, Stone J, Embree G, Paccagnella A, Hoang L, Galanis E. Salmonella enterica servoar Agbeni, British Columbia, Canada, 2011. Emerg Infect Dis. 2012; 18(9):1542-3.

(7) Rounds JM, Hedberg C, Meyer S, Boxrud DJ, Smith KE. Salmonella enterica PFGE clusters, Minnesota, USA, 2001-2007. Emerg Infect Dis. 2010; 16(11):1678-85.

(8) Rounds JM, Boxrud DJ, Jawahir SL, Smith KE. Dynamics of Escherichia coli O157:H7 outbreak detection and investigation, Minnesota 2000-2008. Epidemiol Infect. 2012; 140(8):1430-8.

(9) Gaulin C, Currie A, Gravel G, Hamel M, LeBlanc MA, Ramsay D, Bekal S. Summary of 11 years of enteric outbreak investigation and criteria to initiate an investigation, Province of Quebec, 2002 through 2012. JFP. Sous presse 2014.

\section{Remerciements}

Les auteurs tiennent à remercier les représentants de la Fraser Health Authority, de l'Interior Health Authority, de la Northern Health Authority, de la Vancouver Coastal Health Authority et de la Vancouver Island Health Authority pour leur appui à ce travail et leur collaboration au cours des enquêtes sur les éclosions ainsi que les laboratoires de microbiologie clinique de la Colombie-Britannique et le Laboratoire de santé publique et de référence en microbiologie de la Colombie-Britannique responsables du diagnostic des infections entériques.

\section{Conflit d'intérêts}

II n'y a aucun conflit d'intérêts à déclarer.

\section{Financement}

s.o. 